\title{
Modeling Structures of Open Cell Foams
}

\author{
Zhengwei $\mathrm{Nie}^{1 *}$, Yuyi $\operatorname{Lin}^{1}$, Qingbin Tong ${ }^{2}$
}

\begin{abstract}
This work proposes an original geometrical model based on randomly packed spheres using Laguerre-Voronoi tessellations to simulate geometrical and topological characteristics in the microstructure of open cell foams. The model can be used to analyze the effect of coefficient of variation on the pores distribution in real foams. The distribution of foam-cell volumes in foam structures generated in this work is dependent on the log-normal distribution of sphere volumes in corresponding randomly packed spheres. The statistical data of modeled foam structures, including distribution of the cell volume, face and edge number is very close to the characteristics of real materials. The results also show that a higher coefficient of variation in the sphere diameter would decrease the average number of faces per cell. The average number of faces varies from 13.56 to 14.43 for different coefficients of variation of sphere diameter, while the average number of faces in the Poisson-Voronoi tessellation structures is approximately 15.5 . Furthermore, the porosity of foam structures, $\varepsilon$, decreases with the ratio of strut diameter to the average diameter of randomly packed spheres, $d_{s} / E(d)$, while the specific surface area of foams, $S_{V}$, increases with $d_{s} / E(d)$.
\end{abstract}


Keywords: open cell foams, Laguerre-Voronoi tessellation, randomly packed spheres, structure modeling

\section{Introduction}

Open cell foams: ceramic, metal, or polymer, have been used in a wide range of industrial areas since they typically possess high specific surface area, high porosity, and efficient thermal insulation [1, 2]. In biomedical applications, open cell foams can be viewed as the scaffolds in implantations to reconstruct bones of human body [3, 4]. For chemical engineering applications, open cell foams serve as filters, heat exchangers, and catalyst supports due to their strong corrosion resistance and good high temperature resistance [5-7]. Open cell metallic foams are utilized to construct media for heat energy storage since they usually have an enclosed porous structure [8-11].

A number of papers present different models to build open cell foam structures. In general, three approaches exist to model the foam structures. The first method uses the Kelvin cell to obtain the structure. The Kelvin cell, i.e. so-called tetrakaidecahedron in some publications, has fourteen faces (eight hexagonal and six quadrilateral) and twentyfour vertices as shown in Figure 1. Lord Kelvin studied the bubbles packing pattern in foams. Lord Kelvin (1887) stated that the Kelvin cell was the best shape with minimal surface area for packing equal-sized objects together to fill space. This method entails modeling an idealized periodic cell structure as shown in Figure 2. The Kelvin cell was viewed as an optimal reconstruction model of real foam structures for a long period. However, Kelvin's model shows some unusual mechanical behaviors that have not been verified by researchers in real materials [12]. The random disorder in real materials might 
be responsible for those unusual mechanical behaviors [13]. At present some researchers have already claimed that the Kelvin cell cannot obtain certain mechanical properties of real foams due to its lack of randomness, which is an important feature in real foams, and Kelvin's model is anisotropic, which is clearly not the structure of real foams [12, 13].

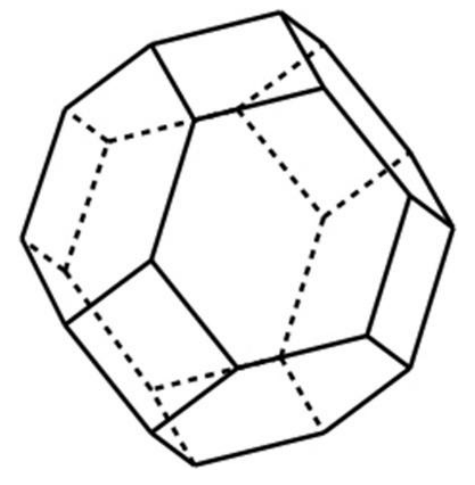

Figure 1 Structure of Kelvin cell

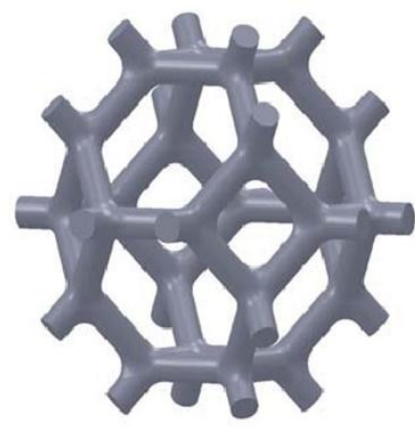

(a)

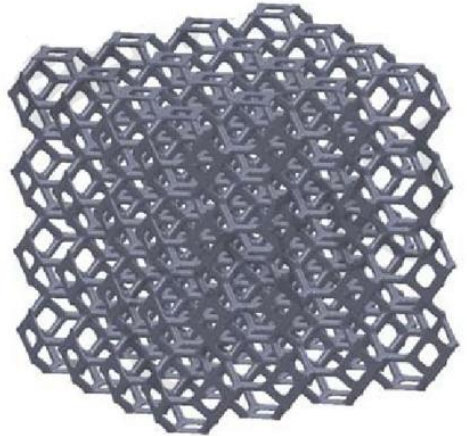

(b)

Figure 2 (a) Single Kelvin cell, and (b) Kelvin multi-cells model

In order to obtain the structure with so-called random disorder, many studies used different methods to consider the randomness of real foam, which are defined as the second approach modeling the foam structures in this work. Habisreuther et al. [14] obtained a randomization of the Kelvin structure by changing the positions of vertices of 
the ordered Kelvin multi-cells structure using vectors with stochastic directions and stochastic values.

Many other researchers generated the random models by Voronoi tessellation, Laguerre-Voronoi tessellation or Poisson-Voronoi tessellation. With this model, random seed points first are set in space and then a cell is generated by defining the space that is closer to its seed point than to any other points. The randomness of this structure is strongly influenced by the spatial distribution of the seed points. However, the number of struts per vertex of the structures generated by Voronoi tessellations is higher than that of real foam structures, and the structural parameters of foam structures obtained by Poisson-Voronoi tessellation is different from real foams [14, 15]. T. Wejrzanowski et al. [15] proposed a three-dimensional model for open cell foams using Laguerre-Voronoi tessellation. They also compared the porosity and surface area with the experimental data. The structures developed by T. Wejrzanowski et al. are closer to real foams than that of the other two tessellations. J. Skibinski et al. [16] reported the validation of the modeling method (Laguerre-Voronoi tessellation) developed in the literature [15] by comparing the pressure drop obtained for modeled structures with the pressure drop of commercial foams and calculated the permeability of open cell foams. The results also state a relationship between pore size distribution and permeability.

The third method uses digitized images or tomographic images of the foams to reassemble the structures of real foams. X-ray micro-tomography $(\mu \mathrm{CT})$ and magnetic resonance imaging (MRI) are very powerful tools that can obtain the architecture and microstructures of materials in a non-destructive and non-invasive way. This approach also is called 3D reconstruction technology, which reconstructs a three-dimensional 
model using two-dimensional image slices such as $\mu \mathrm{CT}$ and MRI stacks [17, 18]. However, three-dimensional models obtained this way are unable to represent all investigated foam structures. Such models are very unique for each individual foam sample. Moreover, because the reconstruction process is high-cost, complicated and timeconsuming, this technology is not always feasible.

To determine foam properties and investigate the effects of geometrical parameters on foam properties, a general statistical foam model is necessary. In this work, a general model is developed which possesses a random spatial structure and is isotropic, but still has a roughly uniform cell size. A non-periodic arrangement of seed points with a pre-selected size distribution is obtained with LAMMPS (an acronym for Large-scale Atomic/Molecular Massively Parallel Simulator). Random foam structures then are generated using Laguerre-Voronoi tessellations. This model could provide the effects of geometrical parameters on the topological properties of foams. To evaluate the generated model in this work, a series of parameters are discussed, such as the average number of faces per foam cell, porosity, and specific surface area, etc.

\section{Methods and modeling}

The model (referred to as the LV foam model) generated in this work is a kind of Laguerre-Voronoi tessellation based on the randomly closed packing of spheres. Voronoi tessellation and Laguerre-Voronoi tessellation are the tools that usually are used to obtain the polycrystalline structures of certain materials or to division spaces in many fields. 


\subsection{Laguerre-Voronoi tessellation}

Laguerre-Voronoi tessellation (LVT) is a kind of weighted Voronoi tessellation [19]. For any point $p_{i}$ in set $S$, a weight $r_{i}$ is provided to get a weight set $r=$ $\left\{r_{1}, r_{2}, \cdots, r_{n}\right\}$, and the distance between $p_{i}$ and any point $q$ is given by:

$$
d_{L}\left(p_{i}, q\right)=\left\{\left[d_{V}\left(p_{i}, q\right)\right]^{2}-r_{i}^{2}\right\}^{1 / 2}
$$

A cell corresponding to point $p_{i}$ is defined as:

$$
v_{L}\left(p_{i}\right)=\left\{p \mid p \in R^{3}, d_{L}\left(p, p_{i}\right)<d_{L}\left(p, p_{j}\right), i \neq j\right\}
$$

and the set of all cells, $V_{L}(S, r)=\left\{v_{l}\left(p_{1}, p_{2}, \cdots, p_{n}\right)\right\}$, is referred to as an LV diagram as shown in Figure 3. Here $v_{l}\left(p_{i}\right)$ is the dominant region of $p_{i}$ with a weight of $r_{i}$. From Figure 3 (c), an LV diagram consists of convex polyhedrons without overlaps interconnected in a topological manner.

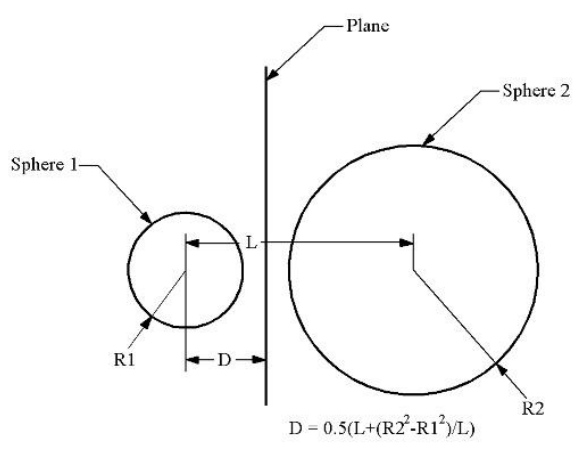

(a)

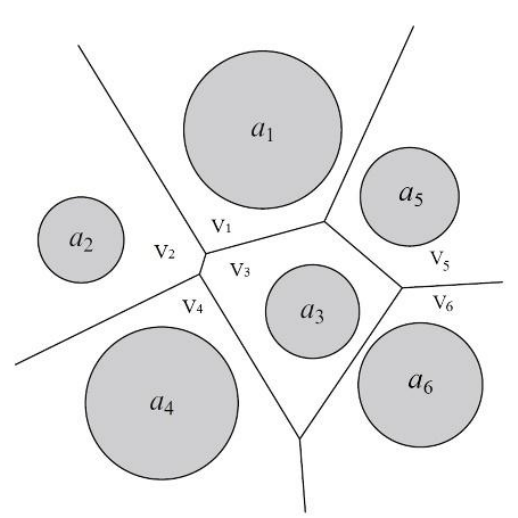

(b) 


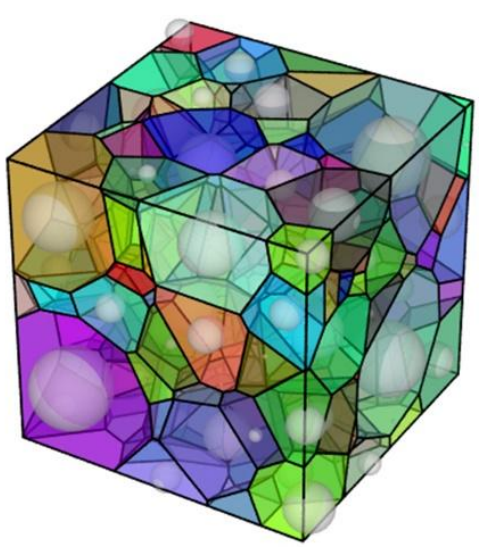

(c)

Figure 3 (a) Schematic illustration of LV tessellation, (b) A 2D, and (c) a 3D Laguerre-Voronoi diagram

\subsection{Randomly packed spheres}

Randomly packed spheres have been extensively studied by both experiments and computer algorithms [20-22]. Among the research methods, computer simulations used to generate randomly packed spheres can be divided into two categories: collective rearrangement algorithms and sequential generation method. In this work, the second method is used and a classical molecular dynamics code, LAMMPS, is employed to generate randomly closed packing of spheres as shown in Figure 4 (visualized and rendered by OVITO). LAMMPS has potential for solid-state materials (metals, semiconductors), soft matter (biomolecules, polymers), and coarse-grained or mesoscopic systems. LAMMPS can be used to model atoms or, more generally, as a parallel particle simulator at the atomic, meso, or continuum scale [23]. 


\subsection{LV tessellation based on randomly packed spheres}

The LV diagram is generated based on randomly packed spheres, which provides a point set, including the centers of spheres and the radii of spheres (Figure 3 (a)). In the LV diagram, each sphere has its own cell, which encloses the whole sphere. Neighboring cells cover adjacent spheres. The sharing face of two cells corresponding to two tangent spheres is just the tangent plane of these two spheres. Therefore, the volume distribution of cells strongly inherits the original spheres' volume distribution. Randomly packed spheres can be regarded as the template in the generation of the LV diagram. In order to improve the computational efficiency, $\mathrm{LV}$ tessellation is programed with $\mathrm{C}++$ and then compiled under a Linux environment in this work. The output files contain a variety of different statistics about the computed LV-cells, including centroid, volume, and the number of vertices, edges, and faces, etc. Also, the program output files include the visualization output for a visualization tool, POV-Ray. An LV diagram based on randomly packed spheres is shown in Figure 5 (visualized and rendered by POV-Ray). 


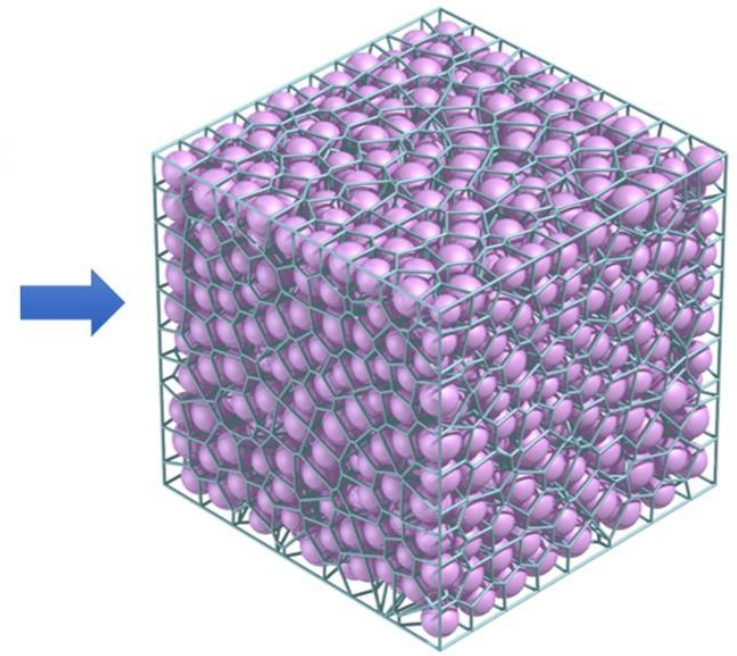

Figure 5 An LV diagram based on randomly packed spheres

\section{$2.4 \quad$ Foam structure generation}

In this work, cells in the LV diagram are assumed to represent individual cells of open cell foams. As shown in Figure 5, foam structure can be obtained by deleting packed spheres, volumes of LV-cells, and interfaces between any two lv-cells and then only retaining the edges and vertices of all LV-cells. To reduce possible boundary effects, cells centered within the outer shell of the LV diagram are cut off and not included in the further statistical procedure as shown in Figure 6. 


\subsection{Modeling procedure}

Due to the randomness and imperfection of foam preparation, real foams usually have a coefficient of variation $(\mathrm{CV})$ of pore volumes in the range of 1.09 to 2.13 and the volume distribution in real foams has been suggested to be log-normal by Rhines et al. $[24,25]$. Therefore, the generation of randomly packed spheres with a defined log-normal distribution of volume in the computations was performed using pre-selected coefficient of variation, $C V(V)$, and average pore volume, $E(V)$.

In conclusion, the process for modeling of open cell foams developed in the present work consists of the following six steps:

(1) Generate random closed packing of more than 1,000 spheres within a cube. Keep the average pore volume $E(V)$ constant, e.g., average pore volume of commercial 10 ppi (pores per inch) alumina foams, and vary the log-normal volume distribution with $C V(V)$, from 0.4 to 3.0 at intervals of 0.2 . Fourteen 
types of randomly packed spheres with different $C V(V)$ values are then obtained.

(2) Repeat the first step three times. In this way, for each type of randomly packed spheres, three groups of more than 1,000 spheres are generated.

(3) Perform Laguerre-Voronoi tessellation to transform each group into an LV diagram.

(4) Delete packed spheres, volumes of LV-cells, and interfaces between any two LV-cells and only keep the edges and vertices of all LV-cells to obtain foam structures.

(5) To reduce possible boundary effect, cells centered within the outer shell of the LV structure are cut off and not included in the further statistical procedure. As a result, each group of foam structures has about 500 effective foam-cells.

(6) For further analysis, merge the three sets of statistical data of the foam structures tessellated based on randomly packed spheres with the same $C V(V)$ value into one.

The process based on the above given six-steps is schematically presented in Figure 7. 
(a)
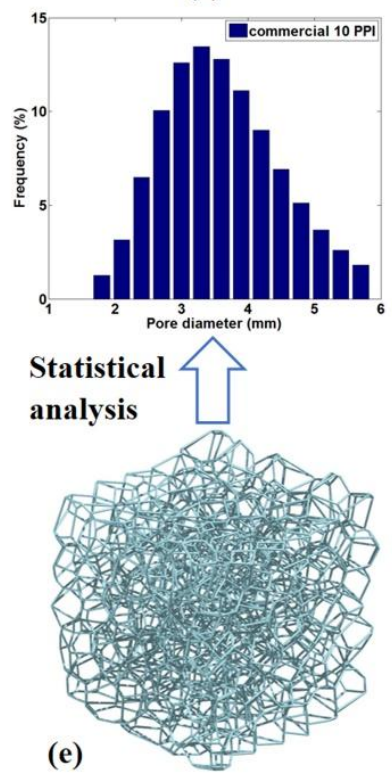
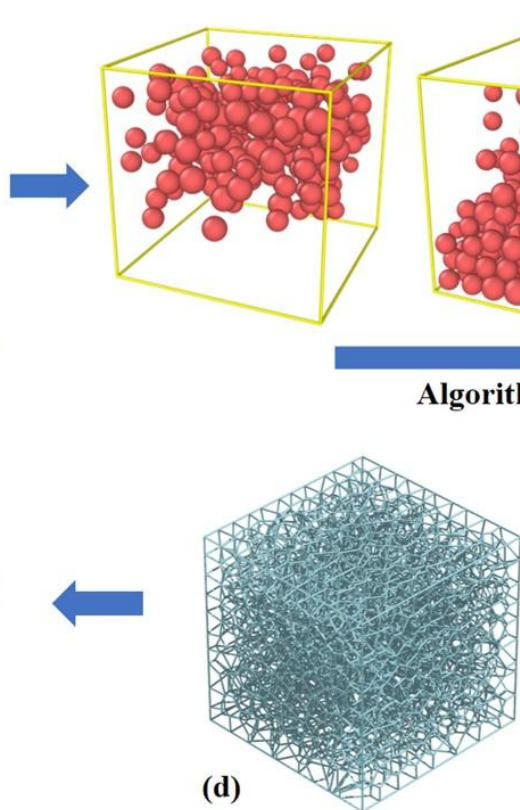

(b)
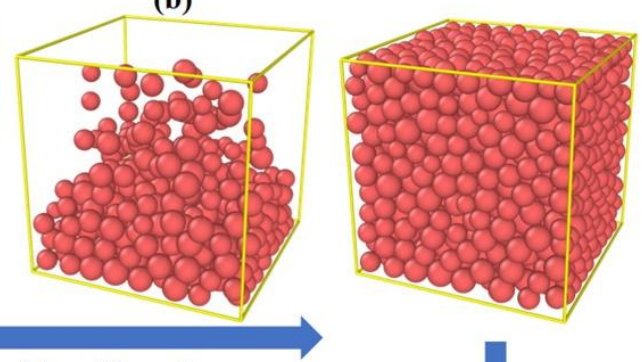

(c)

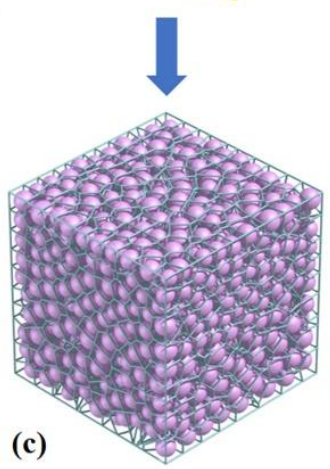

Figure 7 Schematic illustration of the procedure for foam structure generation: (a) spheres of pre-selected volume distribution, (b) the algorithm of randomly packed spheres, (c) LV diagram based on randomly packed spheres, (d) LV-cells with cylindrical edges, and (e) foam structure with cylindrical struts

\section{Results and discussion}

3.1 Distributions of volume, face and edge number

A log-normal distribution has been found to be representative of the features of the structure elements of many natural and engineering materials (Rhines and Patterson [25] and Okazaki and Conrad [24]). Therefore, we adopted the log-normal distribution in this study to analyze statistical data of the foam structures. Its probability density function is described by the following equation:

$$
f(x, \mu \mid \sigma)=\frac{1}{x \sigma \sqrt{2 \pi}} \exp \left(\frac{-(\ln (x)-\mu)^{2}}{2 \sigma^{2}}\right)
$$


where $\mu$ and $\sigma$ are two parameters related to expectation $E(x)$ and variance $\operatorname{Var}(x)$ by equations:

$$
\begin{gathered}
E(x)=e^{\left(\mu+\left(\sigma^{2} / 2\right)\right)} \\
\operatorname{Var}(x)=e^{\left(2 \mu+2 \sigma^{2}\right)}-e^{\left(2 \mu+\sigma^{2}\right)}
\end{gathered}
$$

The coefficient of variation, $C V(x)$, is given by the ratio of the standard deviation $S D(x)$ to the expectation $E(x)$ :

$$
C V(x)=\frac{S D(x)}{E(x)}
$$

where $S D(x)=\operatorname{Var}(x)^{1 / 2}$.

In equations (4) and (5), $E(x)$ and $\operatorname{Var}(x)$ can be calculated by the unbiased estimators $\bar{V}$ and $\frac{1}{n-1} \sum_{i=1}^{n}\left(V_{i}-\bar{V}\right)^{2}$, in which $V_{i}$ is the volume of the $i$ th foam-cell in certain foam structure and $\bar{V}$ is the average volume of cells in corresponding foam structure. In this work, we assume cell volume distribution in each foam structure to be log-normal with parameters defined by equations (4) and (5).

Figure 8 shows the relationship between the standard deviation $(S D)$ and average of foam-cell volumes in the foam structure and $C V(V)$ of spheres volumes in corresponding randomly packed spheres. The mean volume of foam cells decreases linearly with increasing $C V(V)$ of spheres while the standard deviation of foam-cell volumes increases when the $C V(V)$ of spheres increases. The standard deviation changes slightly when the $C V(V)$ of spheres is greater than 1.6. Figure 9, Figure 10, andFigure 11 show the distributions of cell volume and number of cell faces, respectively. Figure 9 includes the histogram of foam-cell volumes in the foam structures and fitted log-normal distribution with corresponding parameters. This foam structure is generated based on 
randomly packed spheres, which have a log-normal volume distribution with $C V(V)=0.4$. Figure 10 shows the histogram of face number per foam-cell in the foam structures and fitted log-normal distribution with corresponding parameters. This foam structure also is generated based on randomly packed spheres with a log-normal volume distribution with $C V(V)=0.4$. Figure 11 shows the histogram of edge number per cell-face in the foam structures and fitted log-normal distribution with corresponding parameters. This foam structure also is generated based on randomly packed spheres with a log-normal volume distribution with $C V(V)=0.4$.

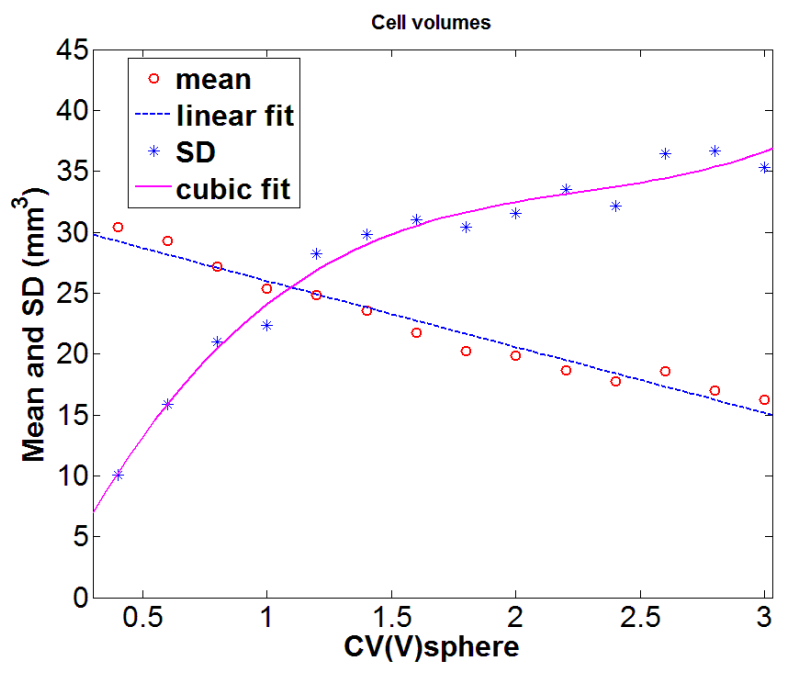

Figure 8 Mean and standard deviation $(S D)$ of foam-cell volumes in the foam structure vs. $C V(V)$ of spheres volumes in corresponding randomly packed spheres 


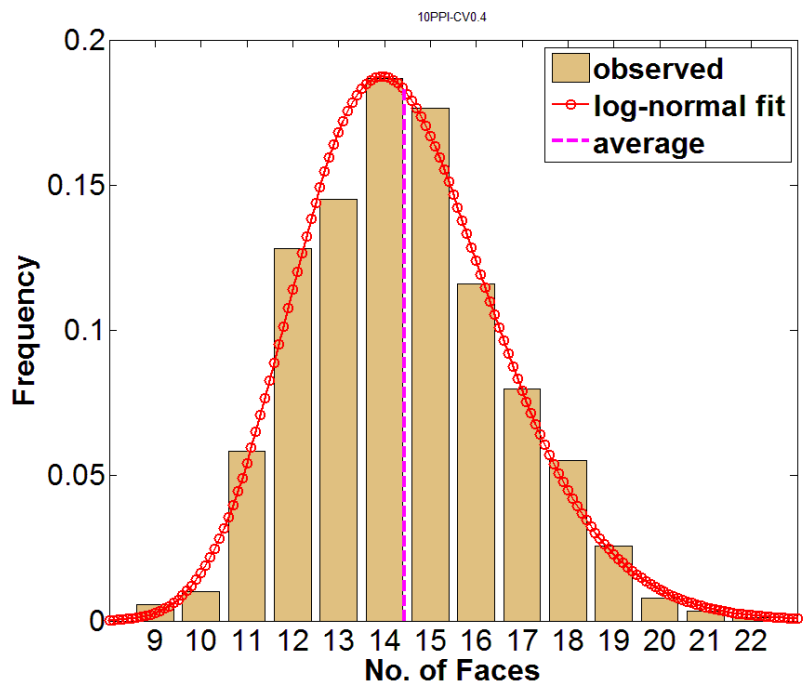

Figure 10 Histogram of face number per foam-cell in foam structures (10 ppi) and fitted log-normal distribution with corresponding parameters; this foam structure is generated based on randomly packed spheres, which have a log-normal volume distribution with $C V(V)=0.4$ 


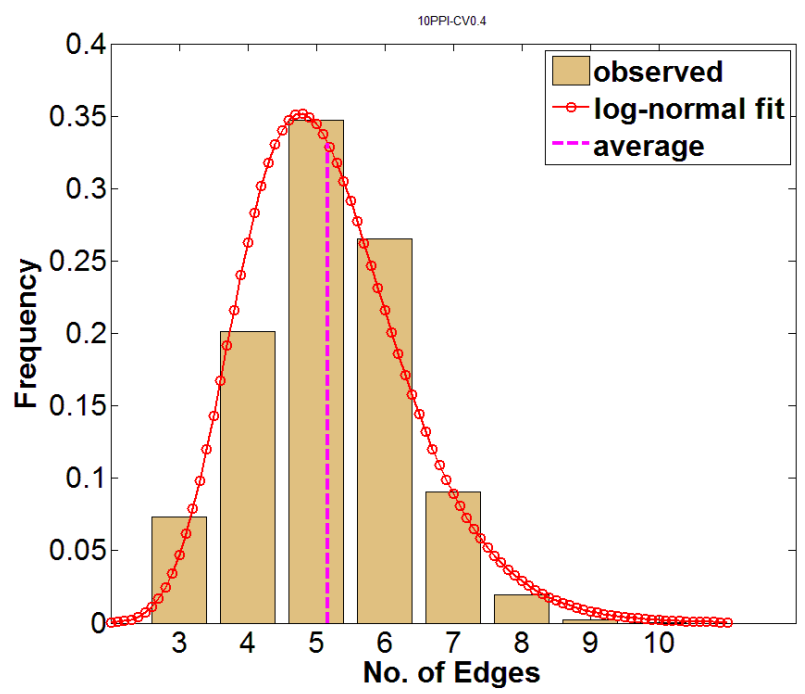

Figure 11 Histogram of edge number per cell-face in foam structures (10 ppi) and fitted log-normal distribution with corresponding parameters; this foam structure is generated based on randomly packed spheres, which have a log-normal volume distribution with $C V(V)=0.4$

\subsection{Effect of cell size inhomogeneity}

For randomly packed spheres with a certain $C V(V)_{\text {sphere }}$ value, the statistics of foam structures, including average number of faces per foam-cell $F$, average number of edges per face $E$, and variation coefficient value of foam-cell volumes distribution $C V(V)_{\text {cell }}$, are obtained. Figure 12 shows the relationship between $F$ and $C V$ of sphere volumes in corresponding randomly packed spheres. Real foam ceramics have the average number of faces per cell in the range of 13.7-15.5 [26]. The results in the authors' work are in good agreement with real foams. The relationship between $E$ and $C V(V)_{\text {sphere }}$ is shown in Figure 13, and between $C V(V)_{\text {cell }}$ and $C V(V)_{\text {sphere }}$ is drawn in Figure 14. Using quadratic fitting, $F$ vs. $C V(V)_{\text {sphere }}$ and $E$ vs. $C V(V)_{\text {sphere }}$ can be expressed as: 


$$
F=0.081\left(C V(V)_{\text {sphere }}\right)^{2}-0.615 C V(V)_{\text {sphere }}+14.669
$$

with a norm of residuals of 0.1082 ,

$$
E=0.0044\left(C V(V)_{\text {sphere }}\right)^{2}-0.0358 C V(V)_{\text {sphere }}+5.1824
$$

with a norm of residuals of 0.0062 . A cubic fit is applied to describe the relationship of $C V(V)_{\text {cell }}$ vs. $C V(V)_{\text {sphere: }}$

$$
\begin{aligned}
C V(V)_{\text {cell }}= & 0.056\left(C V(V)_{\text {sphere }}\right)^{3}-0.395\left(C V(V)_{\text {sphere }}\right)^{2} \\
& +1.492 C V(V)_{\text {sphere }}-0.213
\end{aligned}
$$

with a norm of residuals of 0.1375 .

Figure 12-Figure 14 show that when $C V(V)_{\text {sphere }}$ increases from 0.40 to 3.0, $F$ decreases from 14.43 to $13.56, E$ from 5.17 to 5.11 , while $C V(V)_{\text {cell }}$ increases from 0.33 to 2.17. In addition to good agreement with real ceramic foams, it is worth mentioning here that, for some natural materials, the average number of faces per cell $F$, and the average number of edges per face $E$ are shown in Table 1 . The results also confirm that variation of foam-cell size can be controlled by changing the initial randomly packed spheres size distribution (Figure 14).

Table $1 F$ and $E$ of some natural materials

\begin{tabular}{ccccc}
\hline & No. of cells & $F$ & $E$ & Reference \\
\hline Mixed bubbles & 150 & 13.26 & 5.095 & {$[27]$} \\
Uniform bubbles & 600 & 13.702 & 5.111 & {$[28]$} \\
Vegetable cells & 450 & 13.802 & 5.123 & {$[28]$} \\
\hline
\end{tabular}


Figure 12 Average number of faces per foam-cell in the foam structure vs. $C V$ of sphere volumes in corresponding randomly packed spheres

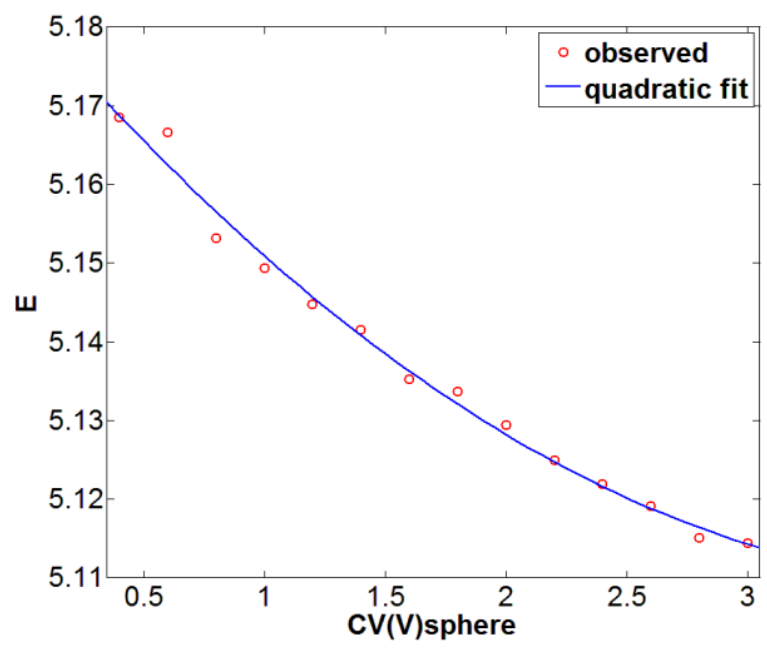

Figure 13 Average number of edges per foam-cell in the foam structure vs. $C V$ of sphere volumes in corresponding randomly packed spheres 


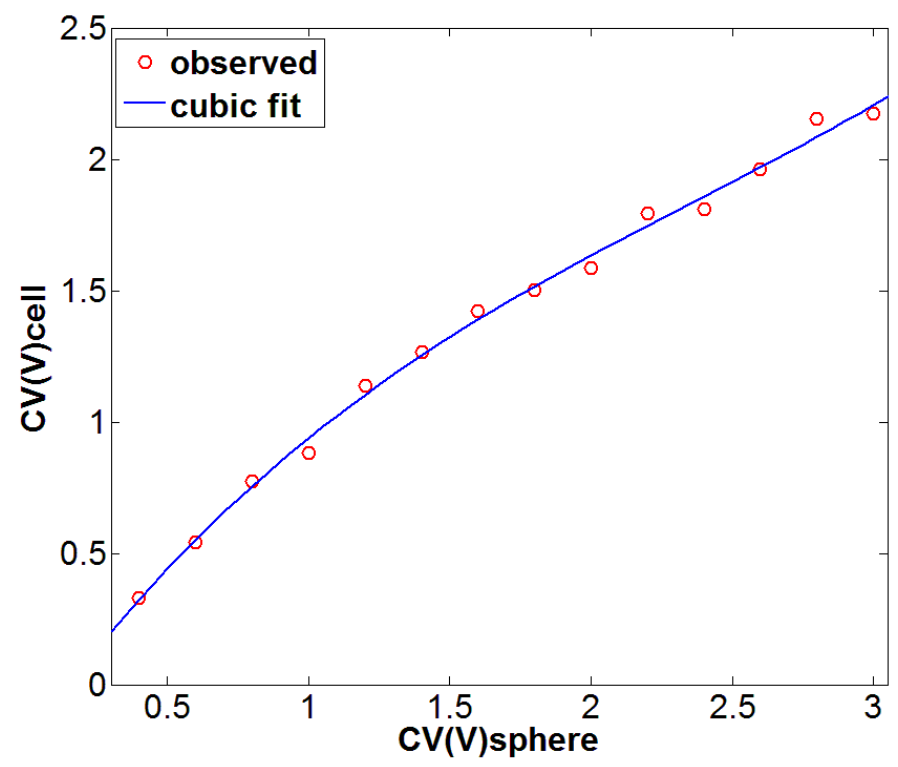

Figure $14 C V$ of foam-cell volume distribution in the foam structure vs. $C V$ of sphere volumes in corresponding randomly packed spheres

\section{Verification}

In this work, the verification of the model is based on work by Gibson and Ashby $[29,30]$. They reported that the average number of struts per cell window is around 5. A dense packed of Kelvin cell (see Figure 15) is discussed here. The Kelvin cell can be viewed as a truncated octahedron, having 8 hexagonal faces and 6 squares. The average number of struts per face is 5.14, which is close to the corresponding value for a pentagonal dodecahedron. Another important geometrical factor which has to be considered is the shape of the strut in the developed foam structures. Most of the published studies simplify the struts to cylinders with a constant diameter along the cell edges of LV diagram. In some work the overlapping struts at vertices is ignored without 
evaluation of the related error [15]. The struts of real foams in this work are simplified by using cylinders of a constant diameter.

Gibson and Ashby [29, 30] proposed the equation below (equation (10)) for the porosity or relative density of a package of Kelvin cells as a function of the cell dimensions:
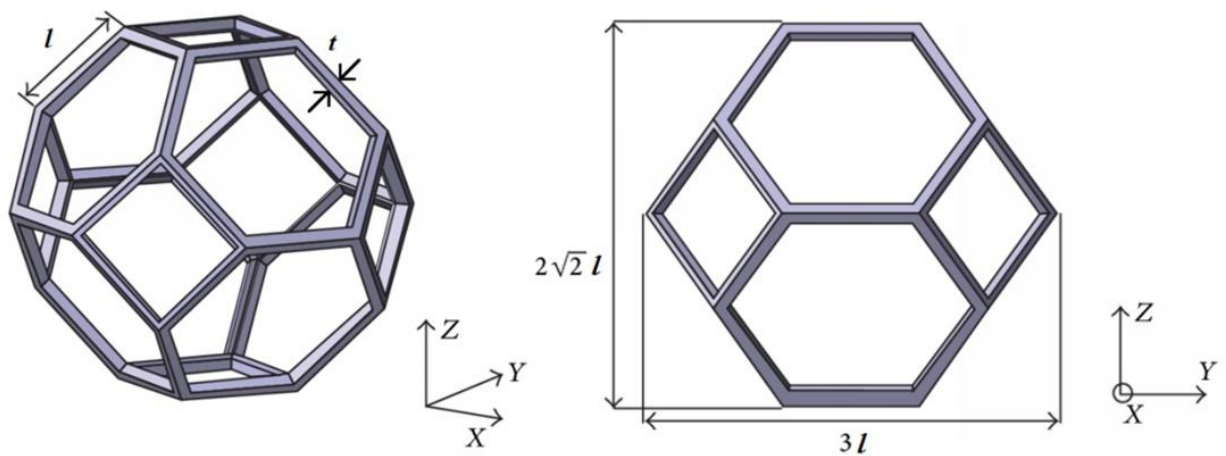

Figure 15 Geometry parameters of single Kelvin cell

$$
\varepsilon=1-\frac{\rho}{\rho_{s}}=1-\varphi=1-1.06\left(\frac{t}{l}\right)^{2}
$$

where $\varepsilon$ is porosity of foam structures; $\rho$ is the density of foam structures, $\rho_{s}$ is the density of solid skeleton and $\rho / \rho_{s}=\varphi$ is called relative density; $l$ is the length and $t$ the edge width of the struts (see Figure $15, t$ can be replaced by $d_{s}$ the diameter of the struts when considering ceramic foams). The length of the struts $l$ is difficult to measure in reality. More conveniently, it can be replaced by another input parameter, the cell diameter $d_{c e l l}$. In the Kelvin cell, the cell diameter $d_{c e l l}$ is defined as the distance between two square faces that are parallel to each other, which can be calculated as follows: 


$$
d_{\text {cell }}=2 \sqrt{2} l
$$

then

$$
\varepsilon=1-1.06\left(\frac{d_{s}}{d_{\text {cell }} / 2 \sqrt{2}}\right)^{2}=1-8.48\left(\frac{d_{s}}{d_{\text {cell }}}\right)^{2}
$$

The specific surface areas of all struts in a Kelvin cell can be obtain through its geometrical parameters. The cell volume is

$$
V_{\text {cell }}=11.31 l^{3}
$$

A unit cell has 36 struts. Each of those belongs to three different cells, which indicates an average of 12 struts per cell. The surface area of one strut (assumed as the cylindrical shape) is equal to $\pi l d_{s}$. This yields a surface area of $12 \pi l d_{s}$ per cell. It can be described in area per unit volume as a function of $d_{s}$ and $d_{\text {cell }}$ :

$$
S_{V}=\frac{12 \pi l d_{s}}{V_{\text {cell }}}=\frac{12 \pi l d_{s}}{11.31 l^{3}}=\frac{12 \pi d_{s}}{11.31\left(d_{\text {cell }} / 2 \sqrt{2}\right)^{2}}=\frac{26.66}{d_{s}}\left(\frac{d_{s}}{d_{\text {cell }}}\right)^{2}
$$

Combining equations (11) and (14) gives the following equation, where the geometric surface area is described as a function of the porosity or relative density and the struts diameter:

$$
\begin{gathered}
S_{V}=\frac{4}{d_{s}}(1-\varepsilon) \\
S_{V}=\frac{4}{d_{s}} \frac{\rho}{\rho_{s}}
\end{gathered}
$$

For dense randomly packed spheres in this work, $d_{c e l l}$ in the equations (12) and (14) can be replaced with the average diameter of packed spheres $E(d)$ :

$$
d_{\text {cell }}=E(d)+2 d_{s}
$$




$$
\begin{gathered}
\varepsilon=1-8.48\left(\frac{d_{s}}{E(d)+2 d_{s}}\right)^{2} \\
S_{V}=\frac{26.66}{d_{s}}\left(\frac{d_{s}}{E(d)+2 d_{s}}\right)^{2}
\end{gathered}
$$

Therefore, the foam structures with different properties, including variation coefficient of foam-cell volumes $C V(V)$, porosity $\varepsilon$, relative density $\rho / \rho_{s}$, and specific surface area $S_{V}$, can be generated by controlling the average diameter $E(d)$ and variation coefficient $C V(d)$ of packed spheres, and the strut diameter $d_{s}$.

Figure 16 shows the porosities of foam structures generated by changing the ratio of strut diameter $d_{s}$ to the average diameter $E(d)$ of randomly packed spheres under $C V(V)=1.5$. The theoretical curve is drawn using equation (18). The results obtained from the model in this current work are agree well with the theoretical result. Using a quadratic expression to fit the relation between porosity vs. $d_{s} / E(d)$ we find

$$
\varepsilon=-6.477\left(d_{s} / E(d)\right)^{2}-0.068\left(d_{s} / E(d)\right)+1.003
$$

with a norm of residuals of 0.0406 .

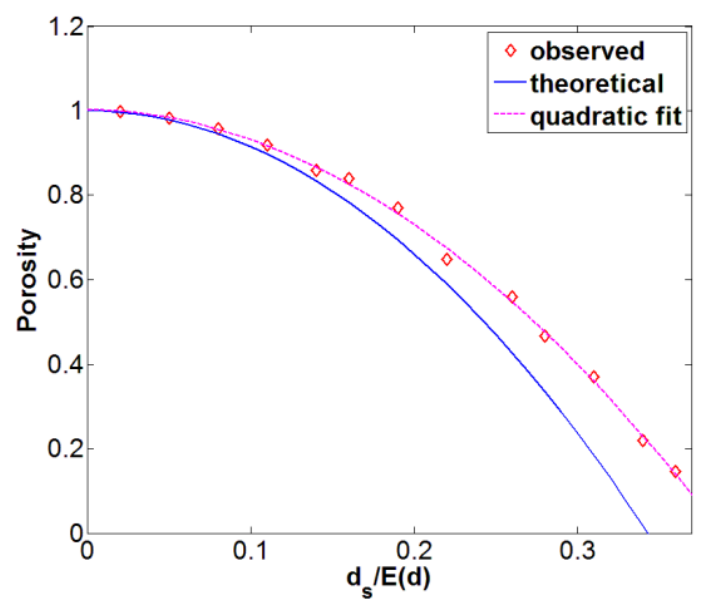

Figure 16 Porosity vs. the ratio of strut diameter $d_{s}$ to the average diameter $E(d)$ of randomly packed spheres under $C V(V)=1.5$ 
Given different strut diameter $d_{s}$, the equation (19) is plot in Figure 17. As presented, using current model different foam structures with different specific surface area can be obtained via changing strut diameter $d_{s}$ and the ratio of $d_{s}$ to the average diameter of randomly packed spheres $E(d)$. Figure 18 shows the observed specific surface areas of foam structures generated in this current work. The simulated results are consistent with the theoretical values.

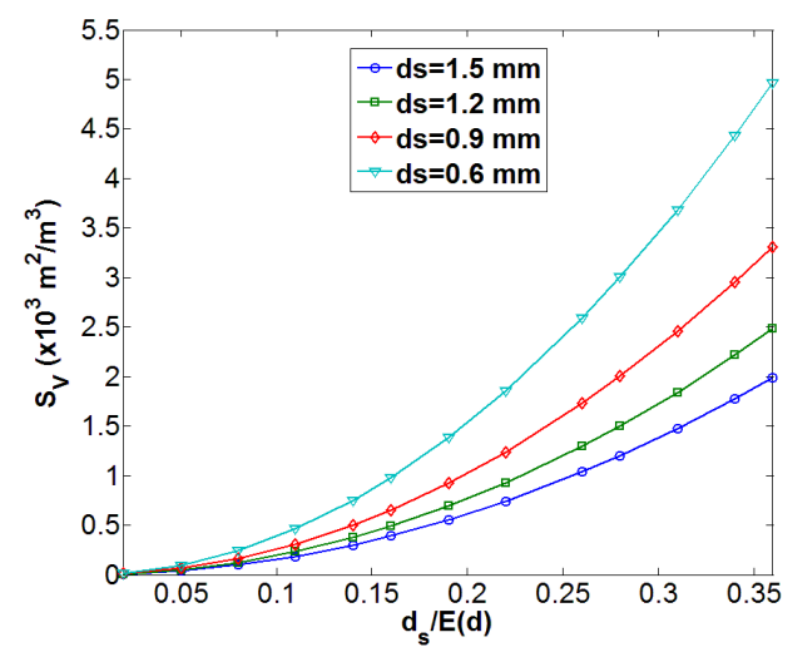

Figure 17 Specific surface area $S_{V}$ vs. $d_{s} / E(d)$ under different strut diameter $d_{s}$

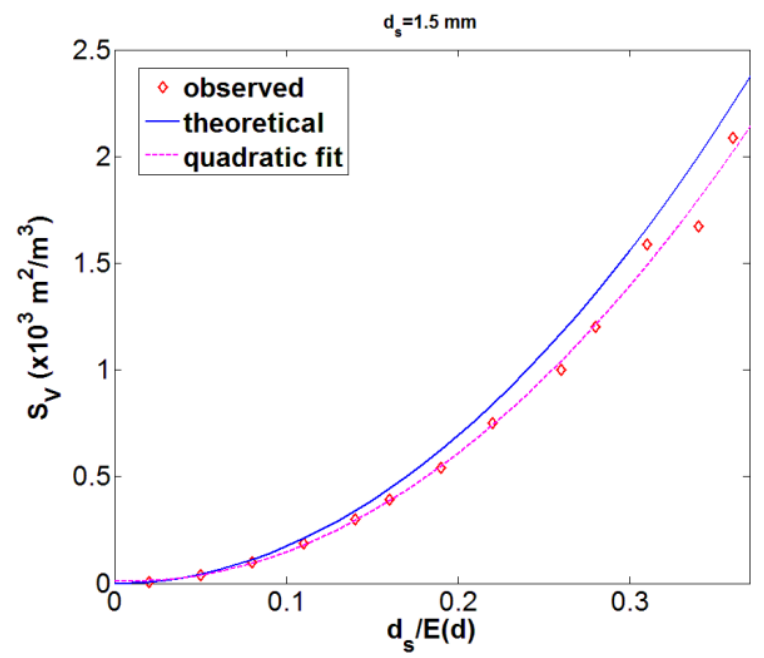

Figure 18 Specific surface area $S_{V}$ vs. $d_{s} / E(d)$ under $d_{s}=1.5 \mathrm{~mm}$ 
The strut diameter $d_{s}$ was set as a variable to investigate its influence on the foam properties for each structure with different $C V(d)$. In total 36 structures were examined with the porosity and specific surface area obtained by equations (18) and (19). The results of these calculations are shown in Figure 19 andFigure 20. Note that when the ratio of the strut diameter to pore size, $d_{s} / E(d)$, is high, $\varepsilon$ and $S_{V}$ are influenced by $C V(d)$ more strongly.

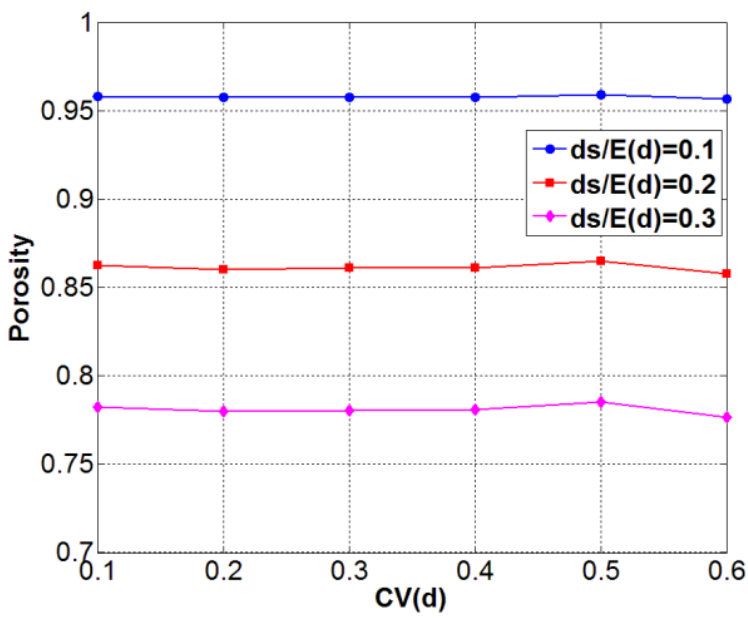

Figure 19 Porosity $\varepsilon$ for simulated foam structures calculated using equation (18)

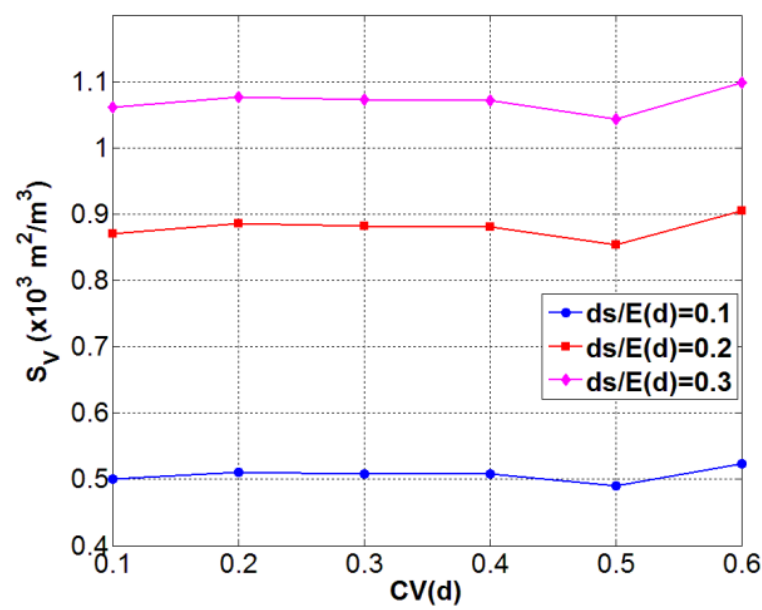

Figure 20 Specific surface area for simulated foam structures calculated using equation (19) 


\begin{tabular}{lccccc}
\hline \multirow{2}{*}{ Parameter } & \multicolumn{5}{c}{ Foam (ppi) } \\
\cline { 2 - 6 } & 10 & 20 & 30 & 40 & 50 \\
\hline Porosity, $\varepsilon(\%)$ & 74 & 69 & 70 & 70 & 75 \\
Specific surface area, $S_{V}\left(\mathrm{~m}^{2} / \mathrm{m}^{3}\right)$ & 1053 & 1476 & 1738 & 2081 & 2449 \\
Average pore diameter, $E(d)(\mathrm{mm})$ & 3.53 & 2.44 & 2.08 & 1.64 & 1.17 \\
Variation coefficient of pore diameter, $C V(d)$ & 0.28 & 0.26 & 0.28 & 0.3 & 0.33 \\
Average strut diameter, $E\left(d_{s}\right)(\mathrm{mm})$ & 1.35 & 1.17 & 0.90 & 0.86 & 0.58 \\
\hline
\end{tabular}

The porosity and specific surface area of foam structures predicted by our model are compared with the data obtained from other models available in the literature as shown in Figure 21 and Figure 22. The real foams analyzed in literature [15] had similar porosity ranging from $69 \%$ to $75 \%$. The simulated results in this work are in a good agreement with that of real foams. The results from other studies presented in Figure 21 shows that porosity differs up to $20 \%$. 


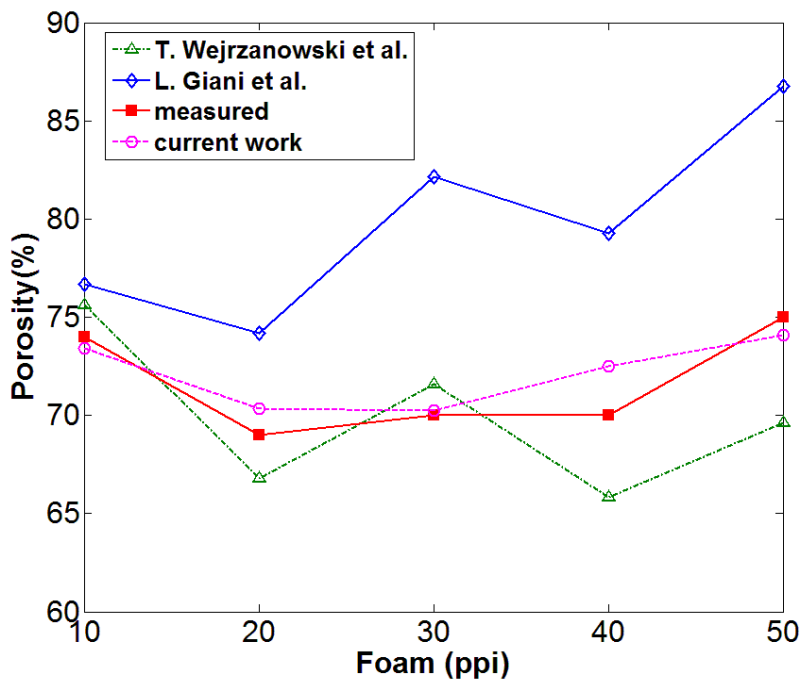

Figure 21 Porosity of foam structures in current work vs. the results in literature

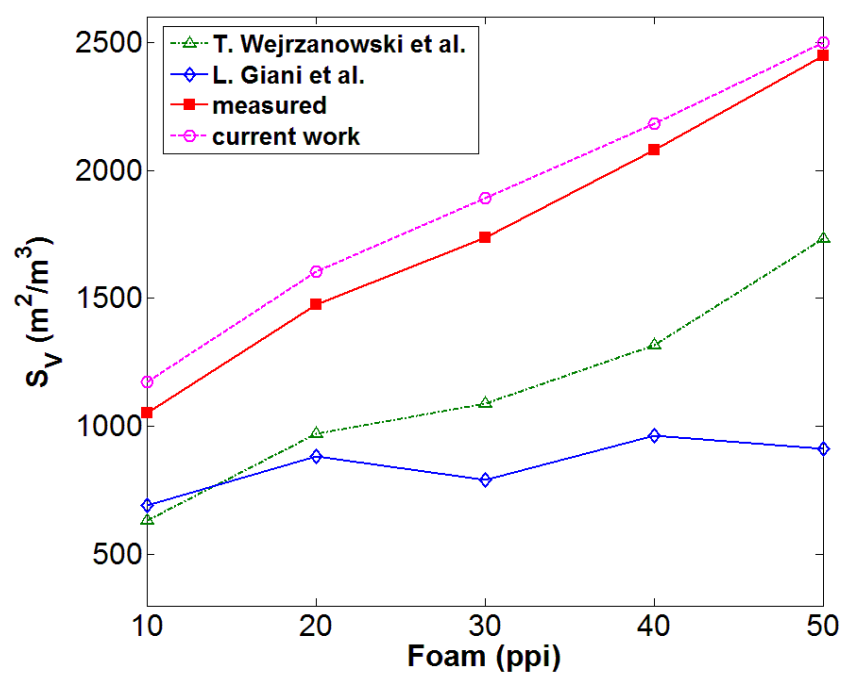

Figure 22 Specific surface area of foam structures in current work vs. the results in literature

Figure 23 shows the cell parameters of real foams modeled in the current work. As shown, the average numbers of vertices, edges, and faces closely agree with those of the Kelvin cell. 


\section{Conclusions}

An original geometrical model based on randomly packed spheres is proposed using Laguerre-Voronoi tessellations, referred to as the LVT structure, to simulate geometrical and topological characteristics in the microstructure of open cell foams. The model can be used to study effects on the pore size in real foams from the coefficient of variation. The distribution of foam-cell volumes in foam structures generated in this work is strongly affected by the log-normal distribution of sphere volumes in randomly packed spheres. The geometrical data of foam structures, including edge number distribution, face distribution, and volume distribution, $E, F$ and $C V(V)_{\text {cell }}$, are very close to those of real materials.

The results also show that higher diversity in the sphere size would decrease the average number of faces per foam-cell, which is between 13.56 and 14.43 for different sphere size variation coefficients. The Laguerre-Voronoi tessellation gives results which 
are much closer to the real foams when compared with the structures generated by Poisson-Voronoi tessellations (number of faces per cell is about 15.5). Further analysis shows that the porosity of foam structures, $\varepsilon$, decreases with the ratio of strut diameter to the average diameter of randomly packed spheres, $d_{s} / E(d)$, while the specific surface area of foams, $S_{V}$, increases with $d_{s} / E(d)$.

Another issue undertaken in this work is the model verification using commercial foams. The model proposed in this work could give much closer results to experimental data compared to other models reported in the literature and give researchers a better understanding of correlations between statistical geometry parameters and the properties of isotropic foam structures.

\section{Acknowledgments}

The authors' special thanks go to Dr. T. Wejrzanowski, a professor at Warsaw University of Technology, who provided insight and expertise that greatly assisted the research. The authors also would like to thank Dr. Chris Rycroft, an assistant professor at Harvard University, for his help in programing of Laguerre-Voronoi tessellations. The authors thank Dr. Peter Hodges for proof reading the manuscript.

\section{REFERENCES}

[1] Dhara, S., et al., Nature inspired novel processing routes for ceramic foams. Advances in applied ceramics, 2005. 104(1): p. 9-21.

[2] Jian-zhong, Z., W. Jiu-Gen, and M. Jia-Ju, Porous structures of natural materials and bionic design. Journal of Zhejiang University Science A, 2005. 6(10): p. 1095-1099.

[3] Schieker, M., et al., Biomaterials as scaffold for bone tissue engineering. European journal of trauma, 2006. 32(2): p. 114-124. 
[4] Swieszkowski, W., et al., Repair and regeneration of osteochondral defects in the articular joints. Biomolecular engineering, 2007. 24(5): p. 489-495.

[5] Placido, E., M. Arduini-Schuster, and J. Kuhn, Thermal properties predictive model for insulating foams. Infrared Physics \& Technology, 2005. 46(3): p. 219231.

[6] Coquard, R. and D. Baillis, Modeling of heat transfer in low-density EPS foams. Journal of heat transfer, 2006. 128(6): p. 538-549.

[7] Kaemmerlen, A., et al., Radiative properties of extruded polystyrene foams: Predictive model and experimental results. Journal of Quantitative Spectroscopy and Radiative Transfer, 2010. 111(6): p. 865-877.

[8] Boomsma, K., D. Poulikakos, and F. Zwick, Metal foams as compact high performance heat exchangers. Mechanics of materials, 2003. 35(12): p. 11611176.

[9] Wang, Q., et al., A review on application of carbonaceous materials and carbon matrix composites for heat exchangers and heat sinks. International Journal of Refrigeration, 2012. 35(1): p. 7-26.

[10] Khateeb, S.A., et al., Thermal management of Li-ion battery with phase change material for electric scooters: experimental validation. Journal of Power Sources, 2005. 142(1): p. 345-353.

[11] Hong, S.-T. and D.R. Herling, Open-cell aluminum foams filled with phase change materials as compact heat sinks. Scripta Materialia, 2006. 55(10): p. 887890.

[12] Zhu, H.X., N.J. Mills, and J.F. Knott, Analysis of the high strain compression of open-cell foams. Journal of the Mechanics and Physics of Solids, 1997. 45(1112): p. 1875-1904.

[13] Roberts, A.P. and E.J. Garboczi, Elastic moduli of model random threedimensional closed-cell cellular solids. Acta materialia, 2001. 49(2): p. 189-197.

[14] Habisreuther, P., N. Djordjevic, and N. Zarzalis, Statistical distribution of residence time and tortuosity of flow through open-cell foams. Chemical Engineering Science, 2009. 64(23): p. 4943-4954.

[15] Wejrzanowski, T., et al., Structure of foams modeled by Laguerre-Voronoi tessellations. Computational Materials Science, 2013. 67: p. 216-221.

[16] Skibinski, J., et al., The influence of pore size variation on the pressure drop in open-cell foams. Materials \& Design, 2015. 87: p. 650-655.

[17] Michailidis, N., et al., An image-based reconstruction of the $3 D$ geometry of an $A l$ open-cell foam and FEM modeling of the material response. Mechanics of Materials, 2010. 42(2): p. 142-147.

[18] Michailidis, N., et al., Experimental and FEM analysis of the material response of porous metals imposed to mechanical loading. Colloids and Surfaces A: Physicochemical and Engineering Aspects, 2011. 382(1-3): p. 124-131.

[19] Fan, Z., et al., Simulation of polycrystalline structure with Voronoi diagram in Laguerre geometry based on random closed packing of spheres. Computational Materials Science, 2004. 29(3): p. 301-308.

[20] Scott, G.D., Packing of spheres: packing of equal spheres. Nature, 1960. 188(4754): p. 908-909. 
[21] Scott, G. and D. Kilgour, The density of random close packing of spheres. Journal of Physics D: Applied Physics, 1969. 2(6): p. 863.

[22] He, D. and N. Ekere, Computer simulation of powder compaction of spherical particles. Journal of materials science letters, 1998. 17(20): p. 1723-1725.

[23] LAMMPS Molecular Dynamics Simulator. 2016; Available from: http://lammps.sandia.gov/.

[24] Okazaki, K. and H. Conrad, Grain size distribution in recrystallized alphatitanium. Transactions of the Japan Institute of Metals, 1972. 13(3): p. 198-204.

[25] Rhines, F. and B. Patterson, Effect of the degree of prior cold work on the grain volume distribution and the rate of grain growth of recrystallized aluminum. Metallurgical Transactions A, 1982. 13(6): p. 985-993.

[26] Roberts, A.P. and E.J. Garboczi, Elastic properties of model random threedimensional open-cell solids. Journal of the Mechanics and Physics of Solids, 2002. 50(1): p. 33-55.

[27] Matzke, E.B. and J. Nestler, Volume-shape relationships in variant foams. A further study of the role of surface forces in three-dimensional cell shape determination. American Journal of Botany, 1946: p. 130-144.

[28] Matzke, E.B., The three-dimensional shape of bubbles in foam-an analysis of the rôle of surface forces in three-dimensional cell shape determination. American Journal of Botany, 1946: p. 58-80.

[29] Gibson, L.J. and M.F. Ashby. The mechanics of three-dimensional cellular materials. in Proceedings of the Royal Society of London A: Mathematical, Physical and Engineering Sciences. 1982. The Royal Society.

[30] Gibson, L.J. and M.F. Ashby, Cellular solids: structure and properties. 1999: Cambridge university press. 
(a)

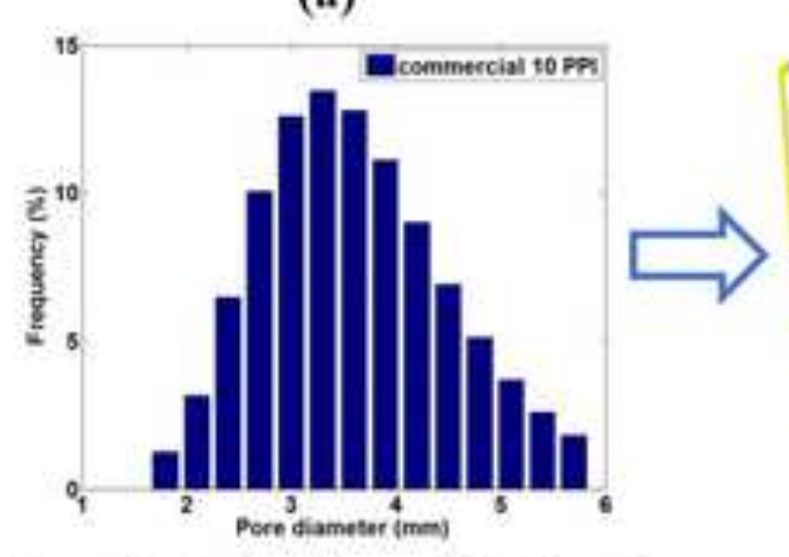

Pre-selected volume distribution

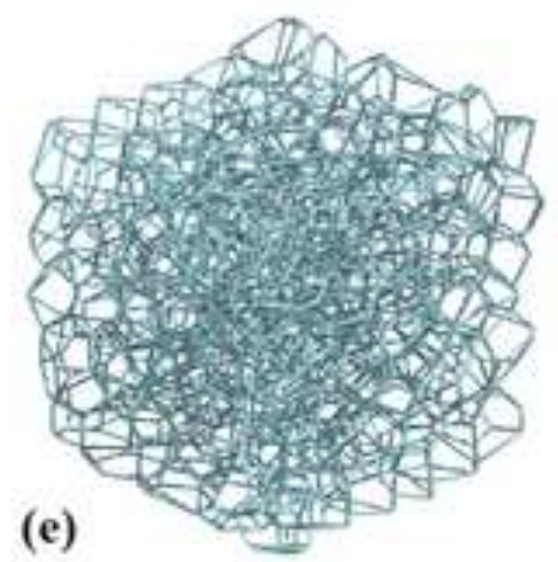

(d) (b)
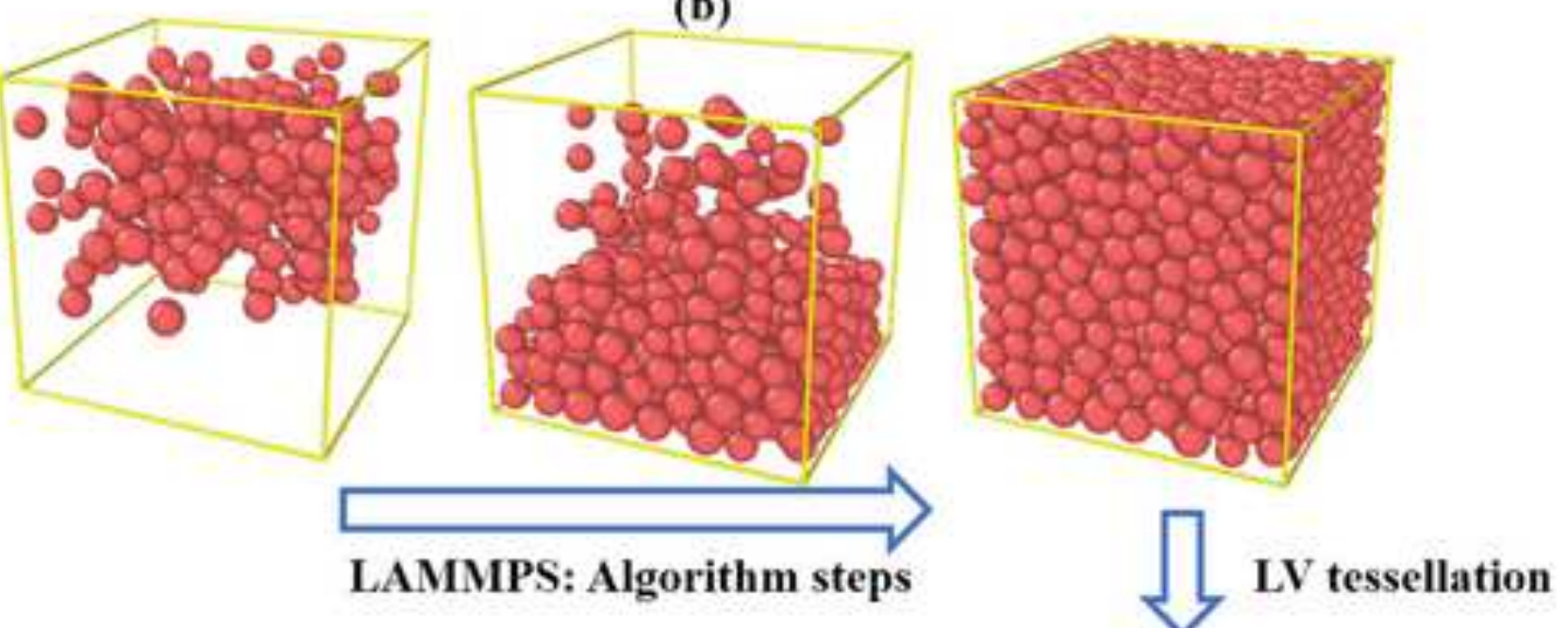

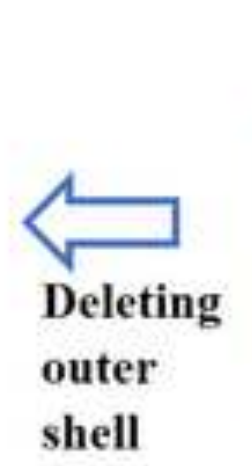

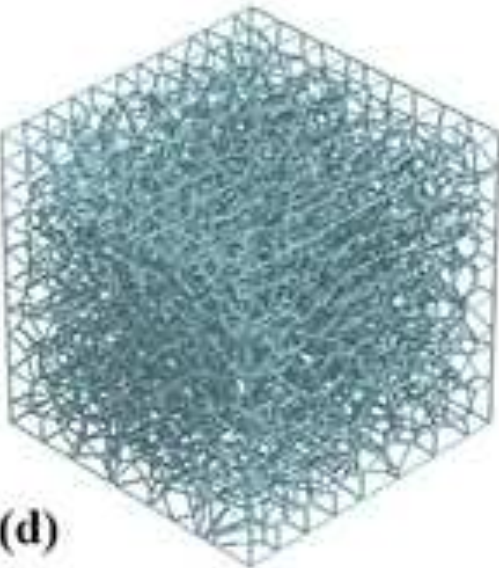
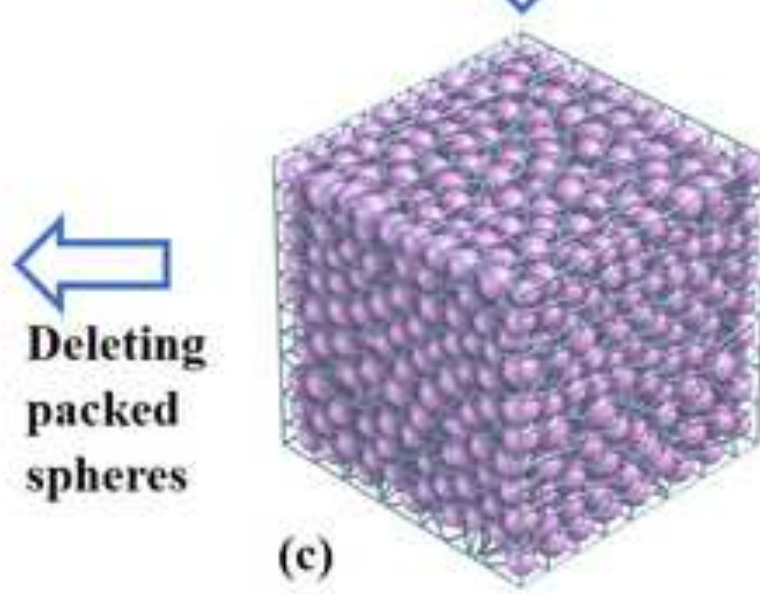

(c) 